\title{
LES JEUX PUBLICITAIRES DANS LES STRATÉGIES DE COLLECTE DE DONNÉES NUMÉRIQUES
}

\author{
Damien RENARD ${ }^{1}$
}

Le jeu publicitaire en ligne est utilisé par les organisations marchandes dans leurs stratégies de collecte de données. Ce qui caractérise ce type de jeu, c'est l'aménagement à l'intérieur du dispositif d'espaces promotionnels dans lesquels le joueur est invité à effectuer une série d'actions non directement liées au jeu. Cette recherche a pour objet de comprendre dans quelle mesure la création d'un environnement ludique est susceptible d'agir sur la décision du joueur de partager des données numériques.. Les résultats d'une étude empirique menée auprès d'un échantillon de consommateurs montrent qu'au-delà du prix à gagner, les joueurs ayant le sentiment de maîtriser le jeu tout en percevant un niveau de défi élevé tendent à partager davantage de données personnelles avec l'organisateur du jeu.

\section{Introduction}

La collecte de données est une étape fondamentale dans l'élaboration des stratégies des organismes publics ou privés. Le marché du Big Data devrait représenter 187 milliards de dollars en 2019

1 Damien Renard est chargé de cours à l'école de communication de l'Université catholique de Louvain.

Recherches en communication, ${ }^{\circ} 49$ - Article publié le 21/03/2019 
(il était évalué à 3,3 milliards en 2010 et 122 milliards en 2015)². Dans le domaine marchand, la collecte de données constitue une opportunité pour personnaliser les offres de biens et services ou encore pour cibler plus efficacement les clients potentiels. Si elle présente un intérêt certain pour les annonceurs, elle peut générer une certaine méfiance de la part des destinataires (Lancelot Miltgen, 2011), dont la décision de fournir des données numériques se base sur un arbitrage entre les bénéfices et les coûts (Li et al., 2010) : si l'accès à un service ou à un produit gratuit peut agir comme un levier, le sentiment de perdre le contrôle ou la perception d'une intrusion dans la vie privée peut freiner l'apport des données personnelles. En ce sens, pour inciter au partage, de nombreuses organisations, essentiellement marchandes, recourent aux jeux-concours en ligne.

Si les jeux-concours sont utilisés en promotion des ventes ${ }^{3}$ depuis les années 1980, ils ont connu un véritable essor avec le développement des technologies numériques et du marketing interactif (Renard \& Darpy, 2017). L'objectif est de capter l'attention de l'internaute et d'influencer sa décision de coopérer avec l'entité organisatrice du jeu. Il s'agit donc, par le jeu, de créer des espaces favorisant le partage d'informations, que ce soit les courriels de ses amis, son âge ou ses centres d'intérêt. Nous nous intéressons ici à l'expérience suscitée par l'inclusion de mécaniques de jeu et aux effets de celle-ci sur la décision de partager des données numériques, réfutant ainsi l'idée de considérer le jeu comme quelque chose de nécessairement improductif (Caillois, 1967). Pour évaluer l'activité de jeu, nous nous appuyons sur la théorie de l'expérience optimale proposée par Csíkszentmihályi (1975). Les résultats de notre étude empirique montrent qu'au-delà du prix à gagner, le partage de données numériques est conditionné par la capacité du jeu publicitaire à offrir une expérience de jeu dans laquelle le joueur puisse valoriser ses compétences et se confronter à un niveau de défi suffisamment élevé.

2 Selon une étude menée par le cabinet IDC (2016) (www.idc.com)

3 Blattberg \& Neslin (1990) en donnent la définition suivante : « la promotion des ventes est un événement marketing centré sur l'action, dont le but est d'avoir un impact direct sur les clients de l'entreprise ». 
Afin de clarifier les enjeux liés au partage des données numériques dans les jeux publicitaires en ligne, plusieurs concepts issus de la littérature sur le sujet sont exposés dans la première partie de cet article. La seconde partie présente les résultats de notre étude menée auprès d'un échantillon de 175 joueurs. Dans la dernière partie, nous concluons sur les implications du partage de données dans les jeux publicitaires en ligne et les futures pistes de recherche suscitées par nos travaux.

\section{Cadre conceptuel}

\subsection{Le jeu promotionnel : un instrument de la communication des entreprises}

Le jeu est un concept complexe et difficile à définir. Si celui-ci a été l'objet d'un nombre important d'études que ce soit par des historiens (Huizinga, 1951), des sociologues (Caillois, 1967) ou des psychologues (Winnicott, 1971), il n'en reste pas moins que la fixation de ses limites reste encore aujourd'hui difficile. Huizinga (1951 [1938], pp. 57-58) le définit comme :

« une activité volontaire accomplie dans certaines limites fixées de temps et de lieu, suivant une règle librement consentie, mais complètement impérieuse, pourvue d'une fin en soi, accompagnée d'un sentiment de tension ou de joie et d'une conscience d'être autrement que dans la vie courante ».

Dans une société marquée par une orientation des individus vers l'émotion ou la séduction (Firat \& Venkatesh, 1995), les annonceurs ont compris leur intérêt à utiliser le jeu dans leurs stratégies de communication. En effet, depuis les années 1980, les jeux et concours ont été un outil privilégié de la communication des entreprises. Ward \& Hill (1991) développent le concept de jeux promotionnels pour désigner l'opportunité pour un ou des consommateurs de se voir attribuer un prix de par leurs chances ou leurs compétences. Alors que les loteries cantonnent le joueur à un rôle de simple spectateur dépendant du hasard, les jeux-concours 
se caractérisent par une attribution mixte du résultat puisque le résultat dépend à la fois du hasard et des compétences du joueur.

\subsection{Le développement des jeux-concours en ligne}

On assiste aujourd'hui à une utilisation croissante des jeux publicitaires en ligne par les entreprises à l'heure où capter l'attention humaine devient un enjeu majeur pour la communication publicitaire. Sur Internet, les annonceurs sont obligés de développer de nouvelles stratégies de communication pour séduire des publics de plus en plus réticents à la publicité. Le jeu publicitaire en ligne (advergame) peut être défini comme une expérience ludique centrée sur l'univers de la marque et/ou de ses produits (Wise et al., 2008). Par exemple, la marque Citroën a lancé en 2016 un jeu sur mobile dans lequel le joueur était invité à participer à une course automobile ${ }^{4}$. Les travaux dans ce domaine ont montré un effet positif, que ce soit sur les attitudes, les intentions ou encore la notoriété (An \& Stern, 2011 ; Panic et al., 2013 ; Van Reijmersdal et al., 2012). Les jeux-concours en ligne s'inscrivent dans cette perspective en combinant l'opportunité pour le joueur de gagner un prix et la participation à une expérience ludique centrée sur l'univers de la marque et/ou de ses produits. Leur utilisation par les organisations marchandes vise deux objectifs : (1) la génération d'une ou plusieurs actions immédiates de la part du joueur et (2) le développement de la notoriété ou de l'image de marque. Ce qui caractérise en effet ce type de jeu, c'est l'aménagement à l'intérieur du dispositif d'un ou de plusieurs espaces promotionnels dans lequel le joueur est invité, en échange d'une récompense, à effectuer une série d'actions non directement liées au jeu (par exemple, renseigner sa date de naissance en échange de chances supplémentaires d'être tiré au sort). Ces éléments non ludiques doivent ainsi être pensés comme une prolongation du jeu dans la mesure où ces actions ont une incidence sur le résultat final. Dans le cadre de cette étude, nous nous intéressons aux effets

4 https://fr-media.citroen.com/fr/citroën/marque/communication/digital/citroën-lance-un-advergame-inédit-à-l'occasion-de-la-nouvelle 
de la participation à un jeu-concours en ligne sur le partage de données numériques. Plus précisément, nous nous centrons sur un type de données : les courriels. La captation de ce type de données autorise en effet l'entité organisatrice à entrer, dans le cadre du jeu, en contact avec la personne dont l'adresse a été renseignée par le joueur.

\section{Les hypothèses de recherche}

\subsection{L'expérience de jeu et le partage de données numériques}

La montée croissante de l'hédonisme dans le champ de la consommation a conduit les organisations à produire des expériences de consommation allant de l'ordinaire à l'extraordinaire (par exemple, la brasserie Heineken Experience à Amsterdam) (Filser, 2002). Pour les organisations, le but recherché à travers la mise en œuvre de ces dispositifs est de favoriser la mise en place d'un contexte expérientiel capable de plonger le participant dans un état de concentration et de plaisir (Carù \& Cova, 2006). Aussi, l'utilisation du jeu s'inscrit dans cette perspective. Pour évaluer la qualité de l'expérience vécue par le joueur, le concept de flow, proposé par Csíkszentmihályi, semble particulièrement adapté. Le flow peut être défini comme :

« un état subjectif dans lequel les personnes sont à ce point absorbées par une activité que plus rien d'autre n'a d'importance en dehors de l'activité elle-même ; l'expérience en elle-même est tellement agréable que les personnes réaliseront l'activité même à un coût élevé, pour le pur intérêt de la réaliser. » (Csíkszentmihályi, 1990, p. 4)

Ce concept a été utilisé pour étudier les activités telles que le sport (Jackson, 1992), les jeux (Csíkszentmihályi, 1975), la musique (O'Neill, 1999) ou encore la navigation en ligne (Hoffman \& Novak, 1996). Il a également été mobilisé dans le contexte des jeux publicitaires en ligne (Steffen et al., 2013). Si la théorie de l'expérience optimale ou du flow a fait l'objet de travaux 
dans des domaines très variés, il existe encore aujourd'hui un débat sur son opérationnalisation (Hoffman \& Novak, 2009). D'un côté, les recherches privilégiant une approche multi-dimensionnelle intègrent un ensemble de caractéristiques, telles que le sentiment de compétence, le contrôle, la téléprésence ou encore la curiosité (Novak et al., 2000). De l'autre côté, certains auteurs défendent une approche uni-dimensionnelle, dérivée du modèle matriciel développé par Csíkszentmihályi (1975). En effet, selon l'auteur, l'état de flow est un état de motivation intrinsèque impliquant un équilibre entre la perception du défi et le sentiment de compétence. Le défi renvoie à l'objectif que l'individu se fixe ou qu'un autre fixe pour lui. Le sentiment de compétence se réfère, de façon générale, aux croyances qu'une personne entretient à propos de ses capacités à organiser et exécuter les actions requises pour gérer les situations " prospectives » (Bandura, 1995). Ceci peut donc influencer ses choix, ses efforts et sa persévérance face aux défis. Le modèle de segmentation en quatre segments met ainsi en avant quatre configurations (Csíkszentmihályi \& Csíkszentmihályi, 1988) :

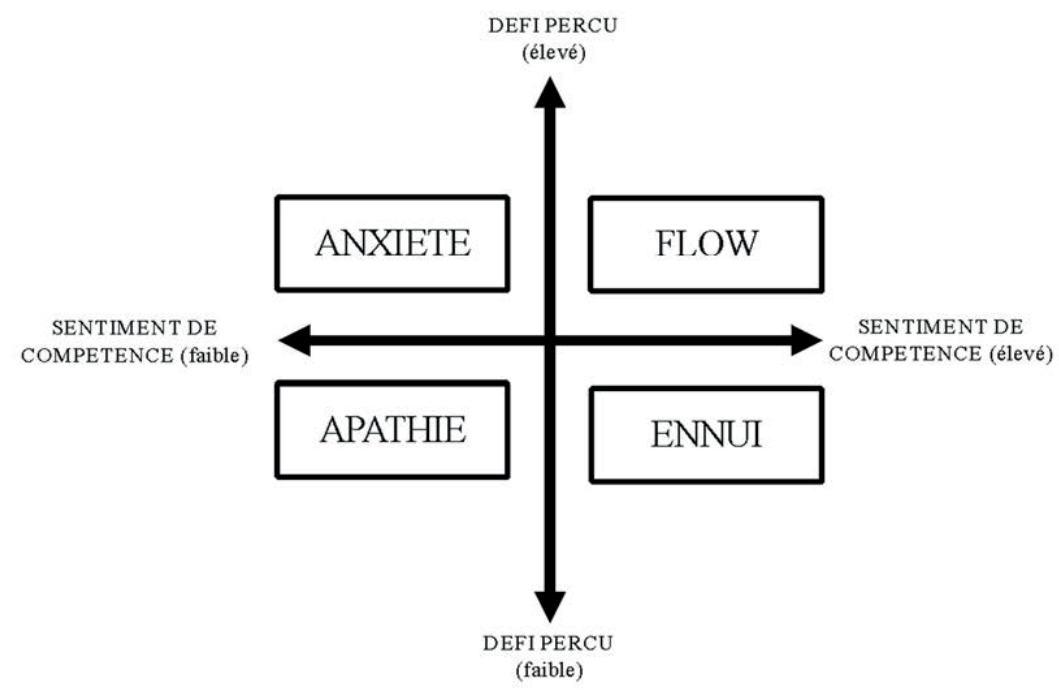

Figure 1. Le modèle de segmentation en quatre segments (adapté de Csíkszentmihályi et Csíkszentmihályi, 1988, p. 261) 
- État de flow : L'individu a le sentiment de se confronter à un véritable défi auquel il estime avoir la capacité de pouvoir répondre.

- État d'ennui : L'individu a le sentiment d'être compétent pour ce type d'activité mais le défi proposé ne correspond pas à ses attentes.

- État d'apathie : L'individu perçoit un niveau de défi faible auquel il estime ne pas avoir la capacité de pouvoir répondre.

- État d'anxiété : L'individu perçoit un niveau de défi auquel il estime ne pas avoir la capacité de pouvoir répondre.

Csíkszentmihályi conditionne ainsi l'expérience optimale ou l'état de flow à la nécessité d'un équilibre entre la perception des défis et l'évaluation de ses propres compétences. Cet état se traduira par un sentiment d'enjouement fort et un degré de concentration élevé (Mathwick \& Rigdon, 2004). Si celui-ci perçoit un déséquilibre entre le niveau d'adversité perçue et ses propres compétences, il peut alors basculer dans un état d'anxiété ou d'ennui. Dans le cadre de cette recherche, nous faisons le choix de nous appuyer sur ce modèle pour étudier la décision de partager des données numériques. Selon Phelps et al. (2004), le degré d'implication dans l'activité constitue un facteur important pour expliquer la décision d'un individu d'en parler à son entourage. Il est donc probable qu'un individu dans cet état sera davantage enclin à partager cette expérience avec son entourage. Ainsi, nous soutenons les hypothèses suivantes :

$\mathbf{H}_{1}$ : L'état du joueur exerce un effet sur le nombre de courriels partagés tel que :

a Le nombre de courriels partagés par les joueurs en état d'ennui est significativement plus faible que le nombre de courriels partagés par les joueurs en état de flow.

b Le nombre de courriels partagés par les joueurs en état d'anxiété est significativement plus faible que le nombre de courriels partagés par les joueurs en état de flow. 
c Le nombre de courriels partagés par les joueurs en état d'apathie est significativement plus faible que le nombre de courriels partagés par les joueurs en état de flow.

\subsection{L'attrait du gain et le partage de données numériques}

Un élément fondamental de la participation à un jeu promotionnel est le prix à gagner (Ward \& Hill, 1991). À l'heure actuelle, il reste un élément important malgré le développement de mécaniques interactives. En matière de partage de données personnelles, Ward et al. (2005) ont montré que les internautes étaient plus enclins à partager dès lors qu'ils avaient le sentiment d'obtenir quelque chose en retour. Dans le domaine du jeu, Jeong et Moon (2014) ont confirmé ce résultat en montrant que la présence de dotations exercerait un effet positif sur l'intention de recommandation. Aussi, nous formulons l'hypothèse qu'un individu attiré par le prix à gagner sera davantage disposé à partager des données numériques :

$\mathbf{H}_{2}$ : L'attrait du gain est positivement associé avec le nombre de courriels partagés.

\section{Méthodologie}

\subsection{Le protocole de l'étude}

Pour tester ces hypothèses, nous avons conduit une étude auprès d'un échantillon de 175 personnes. Les participants sont issus d'une base de données mutualisées d'envoi d'offres promotionnelles par courriel.

Les participants étaient invités à jouer à un jeu créé dans le cadre d'une opération de marketing pour un magazine culturel français. $48,5 \%$ des destinataires ont cliqué sur le lien et 361 ont accepté d'y jouer. Ils découvraient alors cinq univers au sein desquels ils devaient répondre à une question afin de tester leurs connaissances en cinéma, littérature, presse, etc. À chaque fois, ils devaient sélectionner une réponse : si celle-ci était correcte, 
ils obtenaient alors 20 points ; si la réponse sélectionnée était incorrecte, ils n'obtenaient pas de point. Par ailleurs, entre chaque univers, ils avaient la possibilité d'inviter un ami à venir jouer pour gagner 10 points supplémentaires par courriel envoyé. Enfin, après le troisième univers, ils pouvaient cumuler 10 points supplémentaires en communiquant leur âge, leur nom, et leur lieu d'habitation.

Les joueurs obtenaient un score leur permettant de participer à un tirage au sort pour un ou plusieurs prix. Les joueurs ayant un score supérieur à 100 pouvaient participer à un tirage pour gagner un voyage à New York; ceux avec un score supérieur à 20 étaient éligibles pour un coffret de musique (disques). À la fin du jeu, une bannière s'affichait invitant les participants à remplir un questionnaire. Ils étaient interrogés sur leur sentiment de compétence durant le jeu, leur perception du défi, leur degré de concentration et d'enjouement ou encore l'attrait du gain. Par ailleurs, le rapport à la marque était également évalué. Les échelles de mesure utilisées dans le questionnaire sont présentées dans la section suivante.

L'échantillon final comprenait 175 répondants ayant répondu à l'ensemble des questions. $56 \%$ étaient des femmes et l'âge moyen se situe autour de 45 ans, soit un profil similaire aux lecteurs du magazine. $29,7 \%$ ont déclaré avoir acheté le magazine au cours des 12 derniers mois. 52 répondants avaient un score supérieur à 100,111 entre 21 et 99 , et 12 entre 0 et 20 . Le temps moyen de jeu était de 4 minutes et 8 secondes.

\subsection{La qualité des échelles de mesure}

Pour évaluer le défi perçu et le sentiment de compétence, les échelles de Mathwick \& Rigdon (2004) ont été adaptées au contexte de notre étude. Nous avons mesuré l'attitude envers l'organisation en trois items (Mazodier \& Quester, 2010) et l'attrait du gain en un item. Enfin, la qualité de l'expérience de jeu est mesurée via deux construits : la concentration (Mathwick \& Rigdon, 2004) et l'enjouement (Ghani \& Desphande, 1994). La qualité des mesures est reportée dans le tableau 1. Les résultats de l'analyse factorielle confirmatoire indiquent que les échelles sont valides et 
fiables. La validité convergente est vérifiée, les indicateurs présentant une variance moyenne extraite (ou une " average variance extracted »(AVE)) supérieure à 0.5. La fiabilité des échelles est satisfaisante puisque l'ensemble des échelles présentent un alpha de Cronbach supérieur à 0.7 . La validité discriminante est également satisfaisante (Tableau 2).

Tableau 1. Fiabilité et validité convergente des variables d'opérationnalisation

\begin{tabular}{|c|c|c|c|c|}
\hline & AVE & CR & $\alpha$ & \\
\hline Défi perçu & .83 & .93 & .90 & $\begin{array}{l}\text { Ce jeu propose un véritable défi } \\
\text { Jouer à ce jeu m'incite à donner le meilleur } \\
\text { de moi-même } \\
\text { J'ai dû mobiliser toutes mes pensées pour } \\
\text { réussir ce jeu }\end{array}$ \\
\hline $\begin{array}{l}\text { Sentiment de } \\
\text { compétence }\end{array}$ & .74 & .89 & .82 & $\begin{array}{l}\text { Je me considère expert de ce type de jeu } \\
\text { Je sais répondre aux défis proposés par ce } \\
\text { genre de jeu } \\
\text { Je me considère meilleur à ce type de jeu } \\
\text { que la plupart des gens }\end{array}$ \\
\hline Enjouement & .78 & .93 & .90 & $\begin{array}{l}\text { Ordinaire/excitant } \\
\text { Désagréable/agréable } \\
\text { Ennuyeux/intéressant } \\
\text { Déplaisant/plaisant }\end{array}$ \\
\hline $\begin{array}{l}\text { Attrait du } \\
\text { gain }\end{array}$ & - & - & - & Attractif/ Pas attractif \\
\hline Concentration & .78 & .91 & .86 & $\begin{array}{l}\text { J'ai été si impliqué(e) dans le jeu que j'en ai } \\
\text { oublié tout le reste } \\
\text { Ce jeu m'a permis de m'évader du quotidien } \\
\text { Jouer à ce jeu m'a plongé dans un autre } \\
\text { univers }\end{array}$ \\
\hline $\begin{array}{l}\text { Attitude } \\
\text { envers } \\
\text { l'organisation }\end{array}$ & .91 & .97 & .95 & $\begin{array}{l}\{\mathrm{X}\} \text { est un magazine agréable } \\
\{\mathrm{X}\} \text { est un magazine que j'apprécie } \\
\{\mathrm{X}\} \text { est un magazine que j'aime }\end{array}$ \\
\hline
\end{tabular}

Notes : $\alpha=$ alpha de Cronbach ; $\mathrm{CR}=$ composite reliability (fiabilité composite) $\mathrm{AVE}=$ average variance extracted (variance moyenne extraite) 
Tableau 2. Validité discriminante des variables d'opérationnalisation

\begin{tabular}{|c|c|c|c|c|c|c|}
\hline & M & 1 & 2 & 3 & 4 & 5 \\
\hline 1 - Expérience de jeu & 3.70 & .840 & & & & \\
\hline 2 - Défi perçu & 2.76 & .698 & .914 & & & \\
\hline $\begin{array}{l}3 \text { - Sentiment de } \\
\text { compétence }\end{array}$ & 2.91 & .296 & .284 & .848 & & \\
\hline 4 - Attrait du gain & 4.61 & .433 & .354 & .137 & 1.000 & \\
\hline $\begin{array}{c}5-\text { Attitude envers } \\
\text { l'organisation }\end{array}$ & 3.39 & .043 & .024 & .063 & .128 & .957 \\
\hline
\end{tabular}

Le nombre de courriels partagés désigne le nombre de courriels envoyés par les participants à leurs contacts durant le jeu. Dans le cadre de notre recueil de données, 46 (26,3\%) ont envoyé une invitation à jouer et seulement $22(12,7 \%)$ ont saisi plus d'une adresse.

\section{Résultats}

\subsection{La classification des joueurs}

Notre objectif est de regrouper les joueurs en classes selon le sentiment de compétence et la perception du défi. Les méthodes de partition des données permettent de grouper des observations dans des classes de manière à ce que les observations appartenant à la même classe soient plus similaires entre elles qu'à celles appartenant aux autres classes. Les résultats du partitionnement direct sont présentés dans le tableau 3. Les membres du groupe 1 ont le sentiment d'être compétents, mais le défi proposé ne correspond pas à leurs attentes (état d'ennui). À l'inverse, les membres du groupe 3 perçoivent un niveau élevé de défi auquel ils estiment ne pas avoir la capacité de pouvoir répondre (état d'anxiété). Enfin, les membres du groupe 2 perçoivent un faible niveau de 
défi auquel ils estiment ne pas avoir la capacité de répondre (état d'apathie) tandis que ceux du groupe 4 ont un score élevé sur les deux dimensions (état de flow).

Tableau 3. Classification des joueurs

\begin{tabular}{l|cccc}
\hline \multicolumn{1}{l|}{$\begin{array}{c}\text { État d'ennui } \\
\text { (groupe 1) }\end{array}$} & $\begin{array}{c}\text { État } \\
\text { d'apathie } \\
\text { (groupe 2) }\end{array}$ & $\begin{array}{c}\text { État } \\
\text { d'anxiété } \\
\text { (groupe 3) }\end{array}$ & $\begin{array}{c}\text { État de flow } \\
\text { (groupe 4) }\end{array}$ \\
\hline $\mathrm{N}=$ & 38 & 58 & 47 & 32 \\
Sentiment de compétence & 4.09 & 1.58 & 2.52 & 4.75 \\
Défi perçu & 1.64 & 1.64 & 3.70 & 4.48 \\
\hline
\end{tabular}

Afin de vérifier la qualité de notre classification, nous avons comparé la qualité de l'expérience de jeu selon l'état du joueur. Les résultats de l'analyse de variance montrent que la qualité de l'expérience diffère selon l'état du joueur $\left(\mathrm{F}_{(1,174)}=28.18, \mathrm{p}=.00\right)$. Le test de contraste montre une différence significative entre les joueurs en état de flow et ceux en état d'apathie $(\mathrm{c}=-1.85, \mathrm{p}=.00)$, en état d'ennui $(c=-1.93, p=.00)$ ou en état d'anxiété $(c=-0.86$, $\mathrm{p}=.00$ ). Cela signifie donc que la qualité de l'expérience de jeu augmente lorsque le joueur a le sentiment de se confronter à un véritable défi auquel il estime avoir la capacité de pouvoir répondre. Par ailleurs, il est intéressant de noter une différence significative entre les joueurs en état d'anxiété et ceux en état d'apathie ( $\mathrm{c}=$ -1.07, $\mathrm{p}=.00)$ ou en état d'ennui $(\mathrm{c}=-0.99, \mathrm{p}=.00)$.

\subsection{L'état de flow, l'attrait du gain et le partage de données numériques}

Nous avons testé via une régression de poisson les effets de l'état du joueur et de l'attrait du gain sur le nombre de courriels saisis. L'attitude envers l'organisation a été ajoutée comme variable de contrôle. Les résultats sont reportés dans le tableau 4.

Nous observons que le nombre d'adresses saisies diffère significativement selon l'état du joueur. Ainsi, nous constatons 
une différence significative et positive entre les joueurs en état de flow et ceux en état d'apathie $(\mathrm{p}=.00)$, d'ennui $(\mathrm{p}=.03)$ ou d'anxiété $(\mathrm{p}=.01)$. Les hypothèses $\mathrm{H}_{1 \mathrm{a},} \mathrm{H}_{1 \mathrm{~b}}$ et $\mathrm{H}_{1 \mathrm{c}}$ sont donc validées. Notre analyse confirme également l'existence d'un lien significatif entre l'attrait du gain et le nombre d'adresses saisies $(p=.00)$, validant ainsi l'hypothèse $\mathrm{H}_{2}$. Lorsque l'attrait du gain augmente d'un point, 1.23 fois plus de courriels sont partagés par l'utilisateur. L'attitude envers l'organisation exerce un effet significatif $(p=.00)$. Lorsque l'attitude envers l'organisation augmente d'un point, 1.34 fois plus de courriels sont partagés par l'utilisateur.

Tableau 4. Régression de poisson (VD : nombre de courriels partagés)

\begin{tabular}{|c|c|c|c|c|c|c|}
\hline & \multirow[t]{3}{*}{$\beta$} & \multirow[t]{3}{*}{ E.S } & \multirow[t]{3}{*}{$\mathrm{p}$} & \multirow[t]{3}{*}{$\operatorname{Exp}(\beta)$} & \multicolumn{2}{|c|}{ Intervalle de confiance } \\
\hline & & & & & Inférieur & Supérieur \\
\hline \multicolumn{3}{|l|}{ État de flow } & & & & \\
\hline Vs. État d'ennui & -.53 & .25 & $(.03)$ & .58 & .35 & 95 \\
\hline Vs. Etat d'apathie & -2.21 & .00 & $(.00)$ & .10 & .04 & .24 \\
\hline Vs. État d'anxiété & -.57 & .22 & $(.01)$ & .56 & .35 & .88 \\
\hline Attrait du gain & .20 & .06 & $(.00)$ & 1.23 & 1.08 & 1.39 \\
\hline $\begin{array}{l}\text { Attitude envers } \\
\text { l'organisation }\end{array}$ & .29 & .05 & $(.00)$ & 1.34 & 1.19 & 1.51 \\
\hline $\begin{array}{l}\text { Test du rapport des } \\
\text { vraisemblances }\left(\chi^{2}\right)\end{array}$ & 99.77 & & $(.00)$ & & & \\
\hline
\end{tabular}




\section{Discussion, limites et voies de recherches futures}

\subsection{Discussion des résultats}

Cette recherche a pour objet de comprendre dans quelle mesure la création d'un jeu publicitaire en ligne est susceptible d'accaparer celui qui s'y livre et d'orienter sa décision de communiquer des informations à l'organisation. En effet, les recherches sur le partage de données montrent que la décision de l'internaute est largement influencée par le contexte dans lequel celui-ci s'inscrit (Acquisti et al., 2015 ; Bansal et al., 2016).

Nos résultats confirment l'effet significatif de l'attrait du gain, validant ainsi les précédentes recherches dans ce domaine (Ward \& Hill, 1991). Néanmoins, il est intéressant de noter que l'attitude envers l'organisation exerce une influence plus importante sur la décision de partager des données numériques. Au-delà du prix à gagner, nos résultats montrent qu'un joueur dans une situation d'adversité et disposant des compétences pour réussir la tâche est plus enclin à partager des données numériques. L'accès à cet état se traduit par ailleurs par un degré d'enjouement et de concentration plus élevé. Ainsi, lorsque le niveau d'adversité perçue augmente et que le joueur se sent compétent dans l'accomplissement de sa tâche, les joueurs ont une impression de contrôle et le partage de données numériques pourrait apparaître alors comme une opportunité supplémentaire de réduire la part d'aléas. Nos résultats confirment les travaux antérieurs dans le domaine des jeux de hasard. En effet, quand les individus ont l'impression d'avoir un contrôle sur les événements, ils ont tendance à surestimer la probabilité que ces événements se produisent (Langer, 1975 ; Burger, 1991).

\subsection{Apports théoriques}

Alors que le jeu a longtemps été opposé à la réalité productive en ce sens qu'il ne produisait rien et ne visait que le divertissement (Huizinga, 1951 [1938]), il semble nécessaire de dépasser cette vision et de le penser comme un environnement aménagé ayant d'autres finalités que de seulement divertir. Les résultats de 
l'étude montrent ainsi que le recours au jeu permet de légitimer un système de collecte de données. En mélangeant des éléments ludiques et non ludiques à l'intérieur du dispositif, il s'agit de convaincre le joueur que l'activité de partage de données est une partie intégrante du jeu. À l'heure où les organisations peuvent transformer cette donnée brute en une connaissance profitable, on peut estimer que le jeu publicitaire n'a plus seulement vocation à divertir et séduire.

\subsection{Limites et voies de recherche futures}

Ce travail présente certaines limites et ouvre de nouveaux sujets pour de futures recherches. Tout d'abord, nous nous sommes limités à un format de jeu publicitaire : le jeu-concours en ligne. D'autres recherches pourraient être menées, incluant d'autres types de jeux publicitaires. Par ailleurs, nous avons étudié l'expérience de jeu à travers deux variables relatives à l'état de flow. Il serait intéressant de répliquer cette étude expérimentale en intégrant d'autres variables, l'opérationnalisation du flow étant encore aujourd'hui largement débattue dans la littérature. Si nos résultats confirment une corrélation entre l'état du joueur et le degré d'enjouement et de concentration, nous n'avons pas analysé le lien entre ces deux variables et la décision de partager des données. Aussi, il serait intéressant d'explorer cette relation. Par ailleurs, nous avons fait le choix de recourir aux méthodes quantitatives, ce qui implique certaines limites dans l'interprétation des résultats. Le recours à une méthodologie qualitative permettrait de préciser certains liens de causalité.

\section{Références}

Acquisti, A., Brandimarte, L. \& Loewenstein, G. (2015). Privacy and Human Behavior in the Age of Information. Science 347(6221), 509-514. Doi : 10.1126/science. aaa1465.

An, S. \& Stern, S. (2011). Mitigating the Effects of Advergames on Children. Journal of Advertising 40(1), 43-56. Doi : 10.2753/JOA0091-3367400103.

Bandura, A. (1995). Exercise of personal and collective efficacy in changing societies. Dans A. Bandura (Ed.). Self-efficacy in changing societies. New York : Cambridge University Press. 
Bansal, G., Zahedi, F. M. \& Gefen, D. (2016). Do context and personality matter? trust and privacy concerns in disclosing private information online. Information and Management 53(1), 1-21. Doi : 10.1016/j.im.2015.08.001.

Blattberg, R. C. \& Neslin, S. A. (1990). Sales promotion - Concepts, methods, and strategies. Englewood Cliffs, NJ : Prentice Hall.

Burger, J. M. (1991). The effects of desire for control in situations with chance determined outcomes: Gambling behavior in lotto and bingo players. Journal of Research in Personality 25, 196-204. Doi : 10.1016/0092-6566(91)90015-I.

Caillois, R. (1967). Les jeux et les hommes. Paris : Gallimard.

Carù, A. \& Cova, B. (2006). Expériences de consommation et marketing expérientiel. Revue française de Gestion 32(162), 99-114. Doi : 10.3166/rfg.162.99-115.

Csíkszentmihályi, M. (1975). Beyond boredom and anxiety: the experience of play in work and games. San Francisco : Jossey-Bass.

Csíkszentmihályi, M. (1990). Flow: the psychology of optimal experience. New York : Harper \& Row.

Csíkszentmihályi, M. \& Csíkszentmihályi, I. S. (Eds.) (1988). Optimal experience: Psychological studies of Flow in consciousness. New York : Cambridge University Press.

Filser, M. (2002). Le marketing de la production d'expériences : statut théorique et implications managériales. Décisions Marketing 28, 13-22.

Firat, A. \& Venkatesh, A. (1995). Liberatory postmodernism and the reenchantment of consumption. Journal of Consumer Research 32(3), 239-267. Doi : 10.1086/209448.

Ghani, J. A. \& Deshpande, S. P. (1994). Task characteristics and the experience of optimal flow in human-computer interaction. Journal of Psychology 128(4), 381-391. Doi : 10.1080/00223980.1994.9712742.

Hoffman, D. L. \& Novak, T. P. (1996), Marketing in Hypermedia Computer-Mediated Environments: Conceptual Foundations. Journal of Marketing 60 (July), 50-68.

Hoffman, D. L. \& Novak, T. P. (2009). Flow Online: Lessons Learned and Future Prospects. Journal of Interactive Marketing 23(1), 23-34. Doi : 10.1016/j.ntmar.2008.10.003.

Huizinga, J. (1951). Homo ludens. Essai sur la fonction sociale du jeu. Paris : Gallimard.

Jackson, S. A. (1992). Athletes in Flow : A qualitative investigation of Flow states in elite figure skaters. Journal of Applied Sport Psychology 4, 161-180. Doi : 10.1080/10413209208406459.

Jeong, J. \& Moon, S. (2014). Invite Your Friends and Get Rewards: Dynamics of Incentivized Friend Invitation in Kakaotalk Mobile Game. Proceedings of the second ACM conference on online social networks. Dublin, Ireland.

Lancelot Miltgen, C. (2011). Vie privée et marketing : Étude de la décision de fournir des données personnelles dans un cadre commercial. Réseaux 167(3), 131-166. Doi : 10.3917/res.167.0131.

Langer, E. J. (1975). The illusion of control. Journal of Personality and Social Psychology $32,311-328$.

Li, H., Sarathy, R. \& Xu, H. (2010). Understanding situational online information disclosure as a privacy calculus. Journal of Computer Information Systems 51(1), 6271. Doi : 10.1080/08874417.2010.11645450.

Mathwick, C. \& Rigdon, E. (2004). Play, Flow, and the Online Search Experience, Journal of Consumer Research 31, 324-332. Doi : 10.1086/422111. 
Mazodier, M. \& Quester, P. (2010). The Effects of Ambush Marketing Disclosure on Attitudes Towards the Ambusher's Brand. Recherche et Applications en Marketing 25(2), 51-68.

Novak, T. P., Hoffman, D. L. \& Yung, Y. F. (2000). Measuring the Customer Experience in Online Environments: A Structural Modeling Approach. Marketing Science 19(1), 22-42. Doi : 10.1287/mksc.19.1.22.15184.

O'Neill, S. (1999). Flow Theory and the Development of Musical Performance Skills. Bulletin of the Council for Research in Music Education 141, 129-134.

Panic, K., Cauberghe, V. \& De Pelsmacker, P. (2013). Comparing tv ads and advergames targeting children: the impact of persuasion knowledge on behavioural responses. Journal of Advertising 42(2-3), 264-273. Doi : 10.1080/00913367.2013.774605.

Phelps, J. E., Regina, L., Mobilio, L., Perry, D. \& Raman, N. (2004). Viral Marketing or Electronic Word-of-Mouth Advertising: Examining Consumer Responses and Motivations to Pass Along Email. Journal of Advertising Research 44(4), 333-348. Doi : 10.1017/S0021849904040371.

Renard, D. \& Darpy, D. (2017). What makes online promotional games go viral? Comparing the impact of player skills versus incentive rewards on game recommendation. Journal of Advertising Research 57(2), 173-181. Doi : 10.2501/JAR-2017-024.

Steffen, C., Gunnar, M. \& Schramm-Klein, H. (2013). Who is the loser when I lose the game? Does losing an advergame have a negative impact on the perception of the brand? Journal of Advertising 42(2-3), 183-95. Doi: 10.1080/00913367.2013.774598.

Van Reijmersdal, E., Rozendaal, E. \& Buijzen, M. (2012). Effects of prominence, involvement, and persuasion knowledge on children's cognitive and affective responses to advergames. Journal of Interactive Marketing 26, 33-42. Doi : 10.1016/j. intmar.2011.04.005.

Ward, S., Bridges, K. \& Chitty, B. (2005). Do Incentives Matter? An Examination of Online Privacy Concerns and Willingness to Provide Personal and Financial Information. Journal of Marketing Communications 11(1), 21-40. Doi : $10.1080 / 1352726042000263575$.

Ward, J. C. \& Hill, R. P. (1991). Designing effective promotional games: opportunities and problems. Journal of Advertising 20(3), 69-81. Doi : 10.1080/00913367.1991.10673348.

Winnicott, D. W., 1971, Jeu et réalité. L'espace potentiel. Paris : Gallimard.

Wise, K., Bolls, P. D., Kim, H., Venkataraman, A. \& Meyer, R. (2008). Enjoyment of advergames and brand attitudes: the impact of thematic relevance. Journal of Interactive Advertising 9, 27-36. Doi : 10.1080/15252019.2008.10722145. 


\section{(c) (1) $\Theta \Theta$ \\ (c) NC ND Publié sous la licence Creative Commons}

«Attribution - Pas d'Utilisation Commerciale - Pas de Modification 4.0 International» (CC BY-NC-ND) 